\title{
A Study on Ricci Solitons in Kenmotsu Manifolds
}

\author{
C. S. Bagewadi, Gurupadavva Ingalahalli, and S. R. Ashoka \\ Department of Mathematics, Kuvempu University, Shankaraghatta-577 451, Shimoga, Karnataka, India
}

Correspondence should be addressed to Gurupadavva Ingalahalli; gurupadavva@gmail.com

Received 27 December 2012; Accepted 16 January 2013

Academic Editors: R. Farnsteiner and V. S. Matveev

Copyright (C) 2013 C. S. Bagewadi et al. This is an open access article distributed under the Creative Commons Attribution License, which permits unrestricted use, distribution, and reproduction in any medium, provided the original work is properly cited.

We study and obtain results on Ricci solitons in Kenmotsu manifolds satisfying $R(\xi, X) \cdot B=0, B(\xi, X) \cdot S=0, S(\xi, X) \cdot R=0$, $R(\xi, X) \cdot \bar{P}=0$, and $\bar{P}(\xi, X) \cdot S=0$, where $B$ and $\bar{P}$ are C-Bochner and pseudo-projective curvature tensor.

\section{Introduction}

A Ricci soliton is a natural generalization of an Einstein metric and is defined on a Riemannian manifold $(M, g)$. A Ricci soliton is a triple $(g, V, \lambda)$ with $g$ a Riemannian metric, $V$ a vector field, and $\lambda$ a real scalar such that

$$
\mathscr{L}_{V} g+2 S+2 \lambda g=0
$$

where $S$ is a Ricci tensor of $M$ and $\mathscr{L}_{V}$ denotes the Lie derivative operator along the vector field $V$. The Ricci soliton is said to be shrinking, steady, and expanding accordingly as $\lambda$ is negative, zero, and positive, respectively [1]. In this paper, we prove conditions for Ricci solitons in Kenmotsu manifolds to be shrinking, steady, and expanding.

In 1972, Kenmotsu [2] studied a class of contact Riemannian manifolds satisfying some special conditions and this manifold is known as Kenmotsu manifolds. Kenmotsu proved that a locally Kenmotsu manifold is a warped product $I \times{ }_{f} N$ of an interval $I$ and a Kaehler manifold $N$ with warping function $f(t)=s e^{t}$, where $s$ is a nonzero constant. Kenmotsu proved that if in a Kenmotsu manifold the condition $R(X, Y)$. $R=0$ holds, then the manifold is of negative curvature -1 , where $R$ is the curvature tensor of type $(1,3)$ and $R(X, Y)$ denotes the derivation of the tensor algebra at each point of the tangent space.

The authors in [3-7] have studied Ricci solitons in contact and Lorentzian manifolds. The authors in [8] have obtained some results on Ricci solitons satisfying $R(\xi, X) \cdot \widetilde{C}=0$, $P(\xi, X) \cdot \widetilde{C}=0, H(\xi, X) \cdot S=0$ and $\widetilde{C}(\xi, X) \cdot S=0$ and now we extend the work to $R(\xi, X) \cdot B=0, B(\xi, X) \cdot S=0$, $S(\xi, X) \cdot R=0, R(\xi, X) \cdot \bar{P}=0$ and $\bar{P}(\xi, X) \cdot S=0$.

\section{Preliminaries}

An $n$-dimensional differential manifold $M$ is said to be an almost contact metric manifold [9] if it admits an almost contact metric structure $(\phi, \xi, \eta, g)$ consisting of a tensor field $\phi$ of type $(1,1)$, a vector field $\xi$, a 1 -form $\eta$, and a Riemannian metric $g$ compatible with $(\phi, \xi, \eta, g)$ satisfying

$$
\begin{gathered}
\phi^{2}=-I+\eta \otimes \xi, \quad \eta(\xi)=1, \\
\eta \circ \phi=0, \quad \phi \xi=0, \\
g(\phi X, \phi Y)=g(X, Y)-\eta(X) \eta(Y), \quad g(X, \xi)=\eta(X),
\end{gathered}
$$

for all vector fields $X, Y$ on $M$.

An almost contact metric manifold $M(\phi, \xi, \eta, g)$ is said to be Kenmotsu manifold [2] if

$$
\left(\nabla_{X} \phi\right) Y=g(\phi X, Y) \xi-\eta(Y) \phi X
$$

From (3), we have

$$
\nabla_{X} \xi=X-\eta(X) \xi
$$

where $\nabla$ denotes the Riemannian connection of $g$.

In an $n$-dimensional Kenmotsu manifold, we have

$$
\begin{gathered}
\eta(R(X, Y) Z)=g(X, Z) \eta(Y)-g(Y, Z) \eta(X), \\
R(X, Y) \xi=\eta(X) Y-\eta(Y) X
\end{gathered}
$$




$$
\begin{gathered}
R(\xi, X) Y=\eta(Y) X-g(X, Y) \xi, \\
R(\xi, X) \xi=X-\eta(X) \xi,
\end{gathered}
$$

where $R$ is the Riemannian curvature tensor.

Let $(g, V, \lambda)$ be a Ricci soliton in an $n$-dimensional Kenmotsu manifold $M$. From (4) we have

$$
\left(\mathscr{L}_{\xi} g\right)(X, Y)=2[g(X, Y)-\eta(X) \eta(Y)]
$$

From (1) and (9) we get

$$
S(X, Y)=-(\lambda+1) g(X, Y)+\eta(X) \eta(Y) .
$$

The above equation yields that

$$
\begin{gathered}
Q X=-(\lambda+1) X+\eta(X) \xi, \\
S(X, \xi)=-\lambda \eta(X), \\
r=-\lambda n-(n-1),
\end{gathered}
$$

where $S$ is the Ricci tensor, $Q$ is the Ricci operator, and $r$ is the scalar curvature on $M$.

2.1. Example for 3-Dimensional Kenmotsu Manifolds. We consider 3-dimensional manifold $M=\left\{(x, y, z) \in R^{3} ; z \neq 0\right\}$, where $(x, y, z)$ are the standard coordinates in $R^{3}$. Let $\left\{E_{1}, E_{2}\right.$, $\left.E_{3}\right\}$ be linearly independent given by

$$
E_{1}=z \frac{\partial}{\partial x}, \quad E_{2}=z \frac{\partial}{\partial y}, \quad E_{3}=-z \frac{\partial}{\partial z} .
$$

Let $g$ be the Riemannian metric defined by $g\left(E_{1}, E_{2}\right)=g\left(E_{2}\right.$, $\left.E_{3}\right)=g\left(E_{1}, E_{3}\right)=0, g\left(E_{1}, E_{1}\right)=g\left(E_{2}, E_{2}\right)=g\left(E_{3}, E_{3}\right)=1$, where $g$ is given by

$$
g=\frac{1}{z^{2}}(d x \otimes d x+d y \otimes d y+d z \otimes d z) .
$$

The $(\phi, \xi, \eta)$ structure is given by

$$
\begin{gathered}
\eta=-\frac{1}{z} d z, \quad \xi=E_{3}=-z \frac{\partial}{\partial z}, \\
\phi E_{1}=-E_{2}, \quad \phi E_{2}=E_{1}, \quad \phi E_{3}=0 .
\end{gathered}
$$

The linearity property of $\phi$ and $g$ yields that $\eta\left(E_{3}\right)=1, \phi^{2} U=$ $-U+\eta(U) E_{3}, g(\phi U, \phi W)=g(U, W)-\eta(U) \eta(W)$, for any vector fields $U, W$ on $M$. By definition of Lie bracket, we have

$$
\left[E_{1}, E_{2}\right]=0, \quad\left[E_{1}, E_{3}\right]=E_{1}, \quad\left[E_{2}, E_{3}\right]=E_{2} .
$$

Let $\nabla$ be the Levi-Civita connection; with respect to above metric $g$ is given by Koszula formula

$$
\begin{aligned}
2 g\left(\nabla_{X} Y, Z\right)= & X(g(Y, Z))+Y(g(Z, X)) \\
& -Z(g(X, Y))-g(X,[Y, Z]) \\
& -g(Y,[X, Z])+g(Z,[X, Y]),
\end{aligned}
$$

and by virtue of it we have

$$
\begin{array}{lll}
\nabla_{E_{1}} E_{3}=E_{1}, & \nabla_{E_{2}} E_{3}=E_{2}, & \nabla_{E_{3}} E_{3}=0, \\
\nabla_{E_{1}} E_{2}=0, & \nabla_{E_{2}} E_{2}=-E_{3}, & \nabla_{E_{3}} E_{2}=0, \\
\nabla_{E_{1}} E_{1}=-E_{3}, & \nabla_{E_{2}} E_{1}=0, & \nabla_{E_{3}} E_{1}=0 .
\end{array}
$$

Clearly (19) shows that $(\phi, \xi, \eta, g)$ satisfies (2), (3), and (4). Thus $M$ is a Kenmotsu manifold.

It is known that

$$
R(X, Y) Z=\nabla_{X} \nabla_{Y} Z-\nabla_{Y} \nabla_{X} Z-\nabla_{[X, Y]} Z
$$

With the help of (19) and (20), it can be easily verified that

$$
\begin{array}{cc}
R\left(E_{1}, E_{2}\right) E_{2}=-E_{1}, & R\left(E_{1}, E_{3}\right) E_{3}=-E_{1}, \\
R\left(E_{1}, E_{1}\right) E_{1}=0, & R\left(E_{2}, E_{1}\right) E_{1}=-E_{2}, \\
R\left(E_{2}, E_{3}\right) E_{3}=-E_{2}, & R\left(E_{2}, E_{2}\right) E_{2}=0, \\
R\left(E_{3}, E_{1}\right) E_{1}=-E_{3}, & R\left(E_{3}, E_{2}\right) E_{2}=-E_{3}, \\
R\left(E_{3}, E_{3}\right) E_{3}=0 .
\end{array}
$$

From the above expression of the curvature tensor we obtain

$$
\begin{aligned}
S\left(E_{1}, E_{1}\right)= & g\left(R\left(E_{1}, E_{2}\right) E_{2}, E_{1}\right) \\
& +g\left(R\left(E_{1}, E_{3}\right) E_{3}, E_{1}\right)=-2 .
\end{aligned}
$$

Similarly we have

$$
\begin{gathered}
S\left(E_{2}, E_{2}\right)=S\left(E_{3}, E_{3}\right)=-2, \\
\left(\mathscr{L}_{\xi} g\right)\left(E_{i}, E_{i}\right)=2\left[g\left(E_{i}, E_{i}\right)-\eta\left(E_{i}\right) \eta\left(E_{i}\right)\right] .
\end{gathered}
$$

Now by $X=Y=E_{i}$, in (1), where $i=1,2,3$ and by virtue of above equations we get the value of $\lambda$ which is strictly greater than 0 . Thus this is an example of expanding Ricci solitons in Kenmotsu manifolds.

\section{Ricci Soliton in a Kenmotsu Manifold Satisfying $R(\xi, X) \cdot B=0$}

Bochner introduced a Kähler analogue of the Weyl conformal curvature tensor by purely formal considerations, which is now well known as the Bochner curvature tensor [10]. A geometric meaning of the Bochner curvature tensor is given by Blair in [11] by using the Boothby-Wang's fibration. In 1969, Matsumoto and Chūman [12] constructed the notion of CBochner curvature tensor in a Sasakian manifold and studied its several properties. 
The C-Bochner curvature tensor [13] $B$ in $M$ is defined by

$$
\begin{aligned}
B(X, Y) Z=R(X, Y) Z+\frac{1}{n+3} & \times[g(X, Z) Q Y-S(Y, Z) X \\
& -g(Y, Z) Q X+S(X, Z) Y \\
& +g(\phi X, Z) Q \phi Y-S(\phi Y, Z) \phi X \\
& -g(\phi Y, Z) Q \phi X+S(\phi X, Z) \phi Y \\
& +2 S(\phi X, Y) \phi Z+2 g(\phi X, Y) Q \phi Z \\
& +\eta(Y) \eta(Z) Q X-\eta(Y) S(X, Z) \xi \\
& +\eta(X) S(Y, Z) \xi-\eta(X) \eta(Z) Q Y] \\
- & +n-1 \\
& +g(\phi X, Z) \phi Y-g(\phi Y, Z) \phi X \\
+ & +\frac{D}{n+3}[\eta(Y) g(X, Z) \xi-\eta(Y) \eta(Z) X \\
& +\eta(X) \eta(Z) Y-\eta(X) g(Y, Z) \xi] \\
& +g(X, Z) Y-g(Y, Z) X], Y) \phi Z]
\end{aligned}
$$

where $D=(r+n-1) /(n+1)$.

Taking $Z=\xi$ in (24) and using (6), (10), (11), we get

$$
B(X, Y) \xi=\left[1-\frac{\lambda}{n+3}+\frac{4}{n+3}\right][\eta(X) Y-\eta(Y) X] .
$$

Similarly using (5), (10), (11), (12) in (24), we get

$$
\begin{aligned}
\eta(B(X, Y) Z)= & {\left[1-\frac{\lambda}{n+3}+\frac{4}{n+3}\right] } \\
& \times[g(X, Z) \eta(Y)-g(Y, Z) \eta(X)]
\end{aligned}
$$

We assume that the condition $R(\xi, X) \cdot B=0$, then we have

$$
\begin{aligned}
& R(\xi, X) B(Y, Z) W-B(R(\xi, X) Y, Z) W \\
& \quad-B(Y, R(\xi, X) Z) W-B(Y, Z) R(\xi, X) W=0 .
\end{aligned}
$$

Using (7) in (27), we get

$\eta(B(Y, Z) W) X-g(B(Y, Z) W, X) \xi+g(X, Y) B(\xi, Z) W$

$$
\begin{aligned}
& -\eta(Y) B(X, Z) W+g(X, Z) B(Y, \xi) W \\
& -\eta(Z) B(Y, X) W+g(X, W) B(Y, Z) \xi \\
& -\eta(W) B(Y, Z) X=0 .
\end{aligned}
$$

By taking an inner product with $\xi$, we have

$$
\begin{aligned}
\eta(B(Y, Z) W) \eta(X)-g(B(Y, Z) W, X) \\
\quad+g(X, Y) \eta(B(\xi, Z) W)-\eta(Y) \eta(B(X, Z) W) \\
\quad+g(X, Z) \eta(B(Y, \xi) W)-\eta(Z) \eta(B(Y, X) W) \\
\quad+g(X, W) \eta(B(Y, Z) \xi)-\eta(W) \eta(B(Y, Z) X)=0 .
\end{aligned}
$$

By using (25), (26) in (29), we have

$$
\begin{aligned}
& {\left[1-\frac{\lambda}{n+3}+\frac{4}{n+3}\right][g(Y, W) g(Z, X)-g(Z, W) g(Y, X)]} \\
& \quad-g(B(Y, Z) W, X)=0 .
\end{aligned}
$$

In view of (24) in (30), then we have

$$
\left[1-\frac{\lambda}{n+3}+\frac{4}{n+3}\right][g(Y, W) g(Z, X)-g(Z, W) g(Y, X)]
$$

$-g(R(Y, Z) W, X)$

$$
\begin{aligned}
-\frac{1}{n+3}[ & g(Y, W) S(Z, X)-S(Z, W) g(Y, X) \\
& -g(Z, W) S(Y, X)+S(Y, W) g(Z, X) \\
& +g(\phi Y, W) S(\phi Z, X)-S(\phi Z, W) g(\phi Y, X) \\
& -g(\phi Z, W) S(\phi Y, X)+S(\phi Y, W) g(\phi Z, X) \\
& +2 S(\phi Y, Z) g(X, \phi W)+2 g(\phi Y, Z) S(X, \phi W) \\
& +\eta(W) \eta(Z) S(Y, X)-\eta(X) \eta(Z) S(Y, W) \\
& +\eta(Y) \eta(X) S(Z, W)-\eta(W) \eta(Y) S(Z, X)]
\end{aligned}
$$

$$
\begin{aligned}
-\frac{D}{n+3}[ & \eta(X) \eta(Z) g(Y, W)-\eta(W) \eta(Z) g(Y, X) \\
& +\eta(W) \eta(Y) g(Z, X)-\eta(Y) \eta(X) g(Z, W)]
\end{aligned}
$$

$$
+\frac{D+n-1}{n+3}[g(\phi Y, W) g(\phi Z, X)
$$

$$
\begin{aligned}
& -g(\phi Z, W) g(\phi Y, X) \\
& +2 g(\phi Y, Z) g(X, \phi W)]
\end{aligned}
$$

$+\frac{D-4}{n+3}[g(Y, W) g(Z, X)-g(Z, W) g(Y, X)]=0$. 
Taking $X=Y=e_{i}$ in (31) and summing over $i=1,2, \ldots, n$. By virtue of (10), (11), (12), and on simplification, we get

$$
\begin{aligned}
& S(Z, W) \\
& =\left[\frac{-(n+4) \lambda-2 n-3}{n+3}+\frac{-n^{2}-6 n+8}{n+3}-\frac{r}{n+3}\right] g(W, Z) \\
& \quad+\left[\frac{(\lambda+1)(n+4)-2+n}{n+3}+\frac{r+4(n-1)}{n+3}\right] \eta(W) \eta(Z) .
\end{aligned}
$$

Putting $Z=W=\xi$ in (32) and by virtue of (10) and (13), we have

$$
\lambda=(n-1) .
$$

Therefore, $\lambda$ positive that is, the Ricci soliton in Kenmotsu manifold is expanding.

Hence we state the following theorem:

Theorem 1. A Ricci soliton in a Kenmotsu manifold satisfying $R(\xi, X) \cdot B=0$ is expanding.

\section{Ricci Soliton in a Kenmotsu Manifolds \\ Satisfying $B(\xi, X) \cdot S=0$}

The condition $B(\xi, X) \cdot S=0$ implies that

$$
S(B(\xi, X) Y, Z)+S(Y, B(\xi, X) Z)=0 .
$$

By using (10) in (34), we have

$$
\begin{aligned}
\eta(Z) & \eta(B(\xi, X) Y)-(\lambda+1) g(B(\xi, X) Y, Z) \\
& -(\lambda+1) g(Y, B(\xi, X) Z)+\eta(Y) \eta(B(\xi, X) Z)=0,
\end{aligned}
$$

the above equation implies that

$$
\begin{aligned}
{[\eta(Z)} & \eta(B(\xi, X) Y)+\eta(Y) \eta(B(\xi, X) Z)] \\
= & (\lambda+1)[g(B(\xi, X) Y, Z)+g(Y, B(\xi, X) Z)] .
\end{aligned}
$$

By using (24) and (26) in (36), we have

$$
\begin{aligned}
2 \eta(X) & \eta(Y) \eta(Z)\left[1-\frac{\lambda}{(n+3)}+\frac{4}{(n+3)}\right] \\
- & {\left[1-\frac{\lambda}{(n+3)}+\frac{4}{(n+3)}\right] } \\
& \times[g(X, Z) \eta(Y)+g(X, Y) \eta(Z)]=0 .
\end{aligned}
$$

Put $X=Y=\xi$ in (37) then the equation is identically satisfied and we do not get the value for $\lambda$. So, we proceed as follows: Taking $X=Y=e_{i}$ in (37) and summing over $i=1,2, \ldots, n$ and by virtue of $(13)$ and $\eta(Z) \neq 0$ conditions, we obtain

$$
\lambda=n+7 \text {. }
$$

Therefore, $\lambda$ is positive that is Ricci soliton in Kenmotsu manifolds satisfying $B(\xi, X) \cdot S=0$ is expanding.

Hence we can state the following theorem.

Theorem 2. A Ricci soliton in a Kenmotsu manifold satisfying $B(\xi, X) \cdot S=0$ is expanding.

\section{Ricci Soliton in a Kenmotsu Manifold \\ Satisfying $S(\xi, X) \cdot R=0$}

Using the following equations:

$$
\begin{aligned}
& S((X, \xi) \cdot R)(U, V) W \\
&=\left(\left(X \wedge_{S} \xi\right) \cdot R\right)(U, V) W=\left(X \wedge_{S} \xi\right) R(U, V) W \\
&+R\left(\left(X \wedge_{S} \xi\right) U, V\right) W+R\left(U,\left(X \wedge_{S} \xi\right) V\right) W \\
&+R(U, V)\left(X \wedge_{S} \xi\right) W,
\end{aligned}
$$

where the endomorphism $X \wedge_{S} Y$ is defined by

$$
\left(X \wedge_{S} Y\right) Z=S(Y, Z) X-S(X, Z) Y,
$$

we have

$$
\begin{aligned}
& S((X, \xi) \cdot R)(U, V) W \\
&= S(\xi, R(U, V) W) X-S(X, R(U, V) W) \xi \\
&+S(\xi, U) R(X, V) W-S(X, U) R(\xi, V) W \\
&+S(\xi, V) R(U, X) W-S(X, V) R(U, \xi) W \\
&+S(\xi, W) R(U, V) X-S(X, W) R(U, V) \xi .
\end{aligned}
$$

By using the condition $S(\xi, X) \cdot R=0$, and by virtue of (10), (12), we have

$$
\begin{aligned}
-\lambda \eta & (R(U, V) W) X \\
- & {[-(\lambda+1) g(X, R(U, V) W)+\eta(X) \eta(R(U, V) W)] \xi } \\
- & \lambda \eta(U) R(X, V) W \\
- & {[-(\lambda+1) g(X, U)+\eta(X) \eta(U)] R(\xi, V) W } \\
& -\lambda \eta(V) R(U, X) W \\
& -[-(\lambda+1) g(X, V)+\eta(X) \eta(V)] R(U, \xi) W \\
& -\lambda \eta(W) R(U, V) X \\
& -[-(\lambda+1) g(X, W)+\eta(X) \eta(W)] R(U, V) \xi=0 .
\end{aligned}
$$

By taking an inner product with $\xi$ and by virtue of (5), (6), (7), and (8), we have

$$
\begin{aligned}
-(\lambda+1) & \eta(X)[g(U, W) \eta(V)-g(V, W) \eta(U)] \\
+ & \lambda[g(V, W) \eta(X) \eta(U)-g(U, W) \eta(X) \eta(V) \\
& \quad-g(U, X) \eta(V) \eta(W)+g(V, X) \eta(U) \eta(W)] \\
+ & (\lambda+1) g(X, R(U, V) W) \\
+ & (\lambda+1) g(X, U)[\eta(W) \eta(V)-g(V, W)] \\
+ & (\lambda+1) g(X, V)[g(U, W)-\eta(W) \eta(U)] \\
+ & g(V, W) \eta(X) \eta(U)-g(U, W) \eta(X) \eta(V)=0 .
\end{aligned}
$$


Taking $X=U=e_{i}$ and summing over $i=1,2, \ldots, n$, we obtain

$$
\begin{aligned}
& 2(\lambda+1)[g(V, W)-\eta(W) \eta(V)] \\
&+(\lambda+1) S(V, W)-(\lambda+1)(n-1) g(V, W) \\
&+(n-1) \eta(V) \eta(W)=0 .
\end{aligned}
$$

Taking $V=W=\xi$ in (44) and by virtue of (12), (13), we obtain

$$
-\lambda(\lambda+n)=0
$$

This implies either

$$
\lambda=0 \text { or } \lambda=-n .
$$

Therefore for any $\lambda=0$ or $\lambda=-n$ the Ricci soliton in Kenmotsu manifolds satisfying $S(\xi, X) \cdot R=0$ is either steady or shrinking.

Hence we can state the following theorem.

Theorem 3. A Ricci soliton in a Kenmotsu manifold satisfying $S(\xi, X) \cdot R=0$ is either steady or shrinking.

\section{Ricci Soliton in a Kenmotsu Manifolds Satisfying $R(\xi, X) \cdot \bar{P}=0$}

The Pseudo-projective curvature tensor $\bar{P}$ is defined by

$$
\begin{aligned}
\bar{P}(X, Y) Z= & a R(X, Y) Z+b[S(Y, Z) X-S(X, Z) Y] \\
& -\frac{r}{n}\left(\frac{a}{n-1}+b\right)[g(Y, Z) X-g(X, Z) Y],
\end{aligned}
$$

where $a, b \neq 0$ are constants. Taking $Z=\xi$ in (47) and using (6), (10), (11), we get

$$
\bar{P}(X, Y) \xi=\left[a+b \lambda+\frac{r}{n}\left(\frac{a}{n-1}+b\right)\right][\eta(X) Y-\eta(Y) X] .
$$

Similarly using (5), (10), (11), (12) in (47), we get

$$
\begin{aligned}
\eta(\bar{P}(X, Y) Z)= & {\left[a+b(\lambda+1)+\frac{r}{n}\left(\frac{a}{n-1}+b\right)\right] } \\
& \times[g(X, Z) \eta(Y)-g(Y, Z) \eta(X)] .
\end{aligned}
$$

We assume that the condition $R(\xi, X) \cdot \bar{P}=0$, then we have

$$
\begin{aligned}
R(\xi, X) & \bar{P}(U, V) W-\bar{P}(R(\xi, X) U, V) W \\
& -\bar{P}(U, R(\xi, X) V) W-\bar{P}(U, V) R(\xi, X) W=0 .
\end{aligned}
$$

Using (7) in (50), we find

$$
\begin{aligned}
& \eta(\bar{P}(U, V) W) X-g(X, \bar{P}(U, V) W) \xi \\
& \quad-\eta(U) \bar{P}(X, V) W+g(X, U) \bar{P}(\xi, V) W \\
& \quad-\eta(V) \bar{P}(U, X) W+g(X, V) \bar{P}(U, \xi) W \\
& \quad-\eta(W) \bar{P}(U, V) X+g(X, W) \bar{P}(U, V) \xi=0 .
\end{aligned}
$$

By taking an inner product with $\xi$ then we get

$$
\begin{aligned}
& \eta(\bar{P}(U, V) W) \eta(X)-g(X, \bar{P}(U, V) W) \\
& \quad-\eta(U) \eta(\bar{P}(X, V) W)+g(X, U) \eta(\bar{P}(\xi, V) W) \\
& -\eta(V) \eta(\bar{P}(U, X) W)+g(X, V) \eta(\bar{P}(U, \xi) W) \\
& -\eta(W) \eta(\bar{P}(U, V) X)+g(X, W) \eta(\bar{P}(U, V) \xi)=0 .
\end{aligned}
$$

By using (48), (49) in (52), we have

$$
\begin{gathered}
-g(X, \bar{P}(U, V) W)+\left[a+b(\lambda+1)+\frac{r}{n}\left[\frac{a}{n+1}+b\right]\right] \\
\times[g(X, V) g(U, W)-g(X, U) g(V, W)]=0 .
\end{gathered}
$$

In view of (47) in (53), we have

$$
\begin{aligned}
-\operatorname{ag}(X, R(U, V) W) \\
-b[(\lambda+1)\{g(V, X) g(U, W)-g(V, W) g(U, X)\} \\
+\eta(V) \eta(W) g(U, X) \\
\quad-g(V, X) \eta(U) \eta(W)] \\
+[a+b(\lambda+1)] \\
\times[g(X, V) g(U, W)-g(X, U) g(V, W)]=0 .
\end{aligned}
$$

Taking $X=U=e_{i}$ in (54) and summing over $i=1,2, \ldots, n$, and on simplification, we get

$$
a S(V, W)=-a(n-1) g(V, W)-b(n-1) \eta(V) \eta(W) .
$$

Putting $V=W=\xi$ in (55) and by virtue of (12), (13), we get the following equation:

$$
\lambda=\frac{(n-1)(a+b)}{a} .
$$

Since $(a+b) / a \neq 0$ implies that $\lambda>0$, that is, the Ricci soliton in Kenmotsu manifold satisfying $R(\xi, X) \cdot \bar{P}=0$ is expanding, hence we state the following theorem.

Theorem 4. A Ricci soliton in a Kenmotsu manifold satisfying $R(\xi, X) \cdot \bar{P}=0$ is expanding.

\section{Ricci Soliton in a Kenmotsu$$
\text { Manifolds Satisfying } \bar{P}(\xi, X) \cdot S=0
$$

The condition $\bar{P}(\xi, X) \cdot S=0$ implies that

$$
S(\bar{P}(\xi, X) Y, Z)+S(Y, \bar{P}(\xi, X) Z)=0 .
$$


By using (10) in (57), we have

$$
\begin{aligned}
\eta(Z) & \eta(\bar{P}(\xi, X) Y)-(\lambda+1) g(\bar{P}(\xi, X) Y, Z) \\
& -(\lambda+1) g(Y, \bar{P}(\xi, X) Z)+\eta(Y) \eta(\bar{P}(\xi, X) Z)=0,
\end{aligned}
$$

that is,

$$
\begin{aligned}
{[\eta(Z)} & \eta(\bar{P}(\xi, X) Y)+\eta(Y) \eta(\bar{P}(\xi, X) Z)] \\
= & (\lambda+1)[g(\bar{P}(\xi, X) Y, Z)+g(Y, \bar{P}(\xi, X) Z)] .
\end{aligned}
$$

By using (47) and (48) in (59), we have

$$
\begin{aligned}
{\left[a+\frac{r}{n}\right.} & {\left.\left[\frac{a}{n-1}+b\right]\right] } \\
\times & {[2 \eta(X) \eta(Y) \eta(Z)} \\
& \quad-g(X, Z) \eta(Y)-g(X, Y) \eta(Z)]=0 .
\end{aligned}
$$

Put $X=Y=\xi$ in (60); then the equation is identically satisfied and we do not get the value for $\lambda$. So, we proceed as follows: taking $X=Y=e_{i}$, summing over $i=1,2, \ldots, n$, and by virtue of $(13)$ and $\eta(Z) \neq 0$ conditions we obtain

$$
\lambda=\frac{(n-1)^{2}(a-b)}{n[a+b(n-1)]} .
$$

Therefore, if $a=b$ in (61) then $\lambda=0$; that is, Ricci soliton in Kenmotsu manifolds satisfying $\bar{P}(\xi, X) \cdot S=0$ is steady. If $a \neq b$ then either $\lambda>0$ for $a>b$ or $\lambda<0$ for $a<b$, that is, the Ricci soliton in Kenmotsu manifold satisfying $\bar{P}(\xi, X) \cdot S=0$ is expanding or shrinking.

Hence we can state the following theorem.

Theorem 5. A Ricci soliton in a Kenmotsu manifolds satisfying $\bar{P}(\xi, X) \cdot S=0$ is steady for $a=b$, expanding for $a>b$ and shrinking for $a<b$.

\section{References}

[1] B. Chow, P. Lu, and L. Ni, Hamilton's Ricci Flow, vol. 77 of Graduate Studies in Mathematics, American Mathematical Society, Providence, RI, USA, 2006.

[2] K. Kenmotsu, "A class of almost contact Riemannian manifolds," The Tohoku Mathematical Journal, vol. 24, pp. 93-103, 1972.

[3] R. Sharma, "Certain results on $K$-contact and $(k, \mu)$-contact manifolds," Journal of Geometry, vol. 89, no. 1-2, pp. 138-147, 2008.

[4] M. M. Tripathi, "Ricci solitons in contact metric manifolds," http://arxiv.org/abs/0801.4222.

[5] C. Călin and M. Crasmareanu, "From the Eisenhart problem to Ricci solitons in $f$-Kenmotsu manifolds," Bulletin of the Malaysian Mathematical Sciences Society, vol. 33, no. 3, pp. 361368, 2010.

[6] C. S. Bagewadi and G. Ingalahalli, "Ricci solitons in Lorentzian $\alpha$-Sasakian manifolds," Acta Mathematica. Academiae Paedagogicae Nyíregyháziensis, vol. 28, no. 1, pp. 59-68, 2012.
[7] G. Ingalahalli and C. S. Bagewadi, "Ricci solitons in $\alpha$-Sasakain manifolds," ISRN Geometry, vol. 2012, Article ID 421384, 13 pages, 2012.

[8] H. G. Nagaraja and C. R. Premalatha, "Ricci solitons in Kenmotsu manifolds," Journal of Mathematical Analysis, vol. 3, no. 2, pp. 18-24, 2012.

[9] D. E. Blair, Contact Manifolds in Riemannian Geometry, vol. 509 of Lecture Notes in Mathematics, Springer, Berlin, Germany, 1976.

[10] S. Bochner, "Curvature and Betti numbers. II," Annals of Mathematics, vol. 50, no. 2, pp. 77-93, 1949.

[11] D. E. Blair, "On the geometric meaning of the Bochner tensor," Geometriae Dedicata, vol. 4, no. 1, pp. 33-38, 1975.

[12] M. Matsumoto and G. Chūman, "On the $C$-Bochner curvature tensor," TRU Mathematics, vol. 5, pp. 21-30, 1969.

[13] J. S. Kim, M. M. Tripathi, and J. Choi, "On C-Bochner curvature tensor of a contact metric manifold," Bulletin of the Korean Mathematical Society, vol. 42, no. 4, pp. 713-724, 2005. 


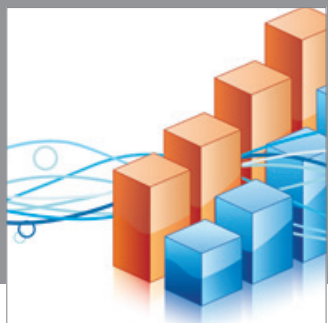

Advances in

Operations Research

mansans

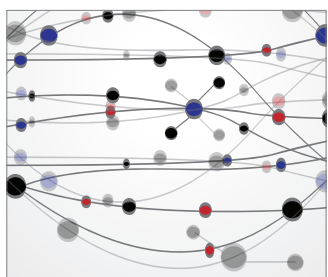

The Scientific World Journal
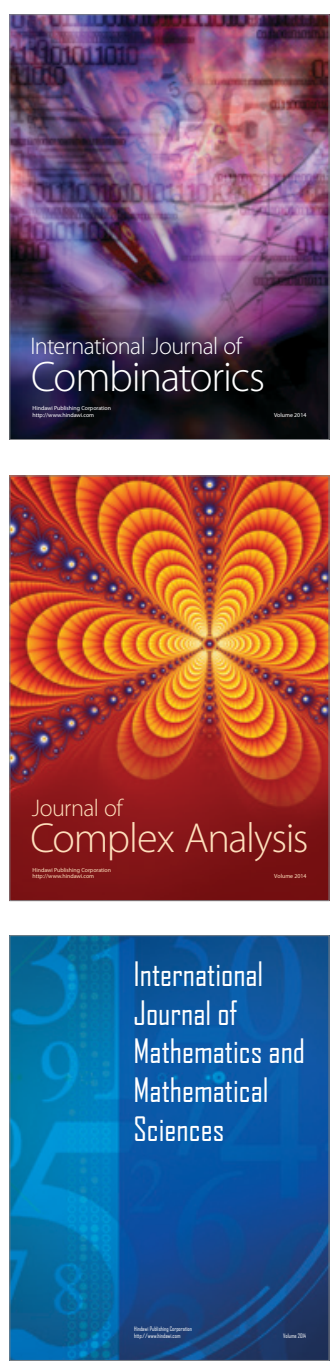
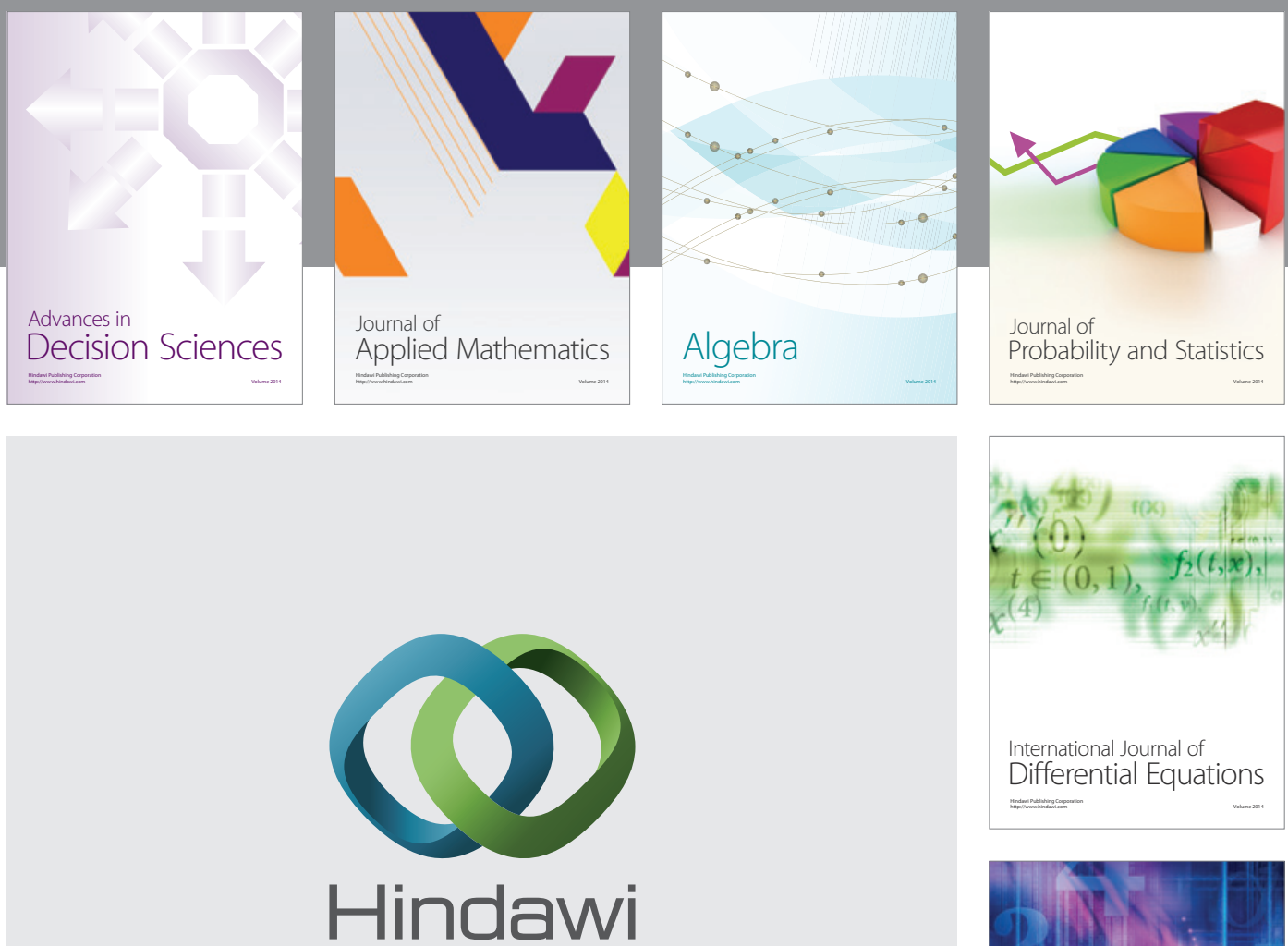

Submit your manuscripts at http://www.hindawi.com
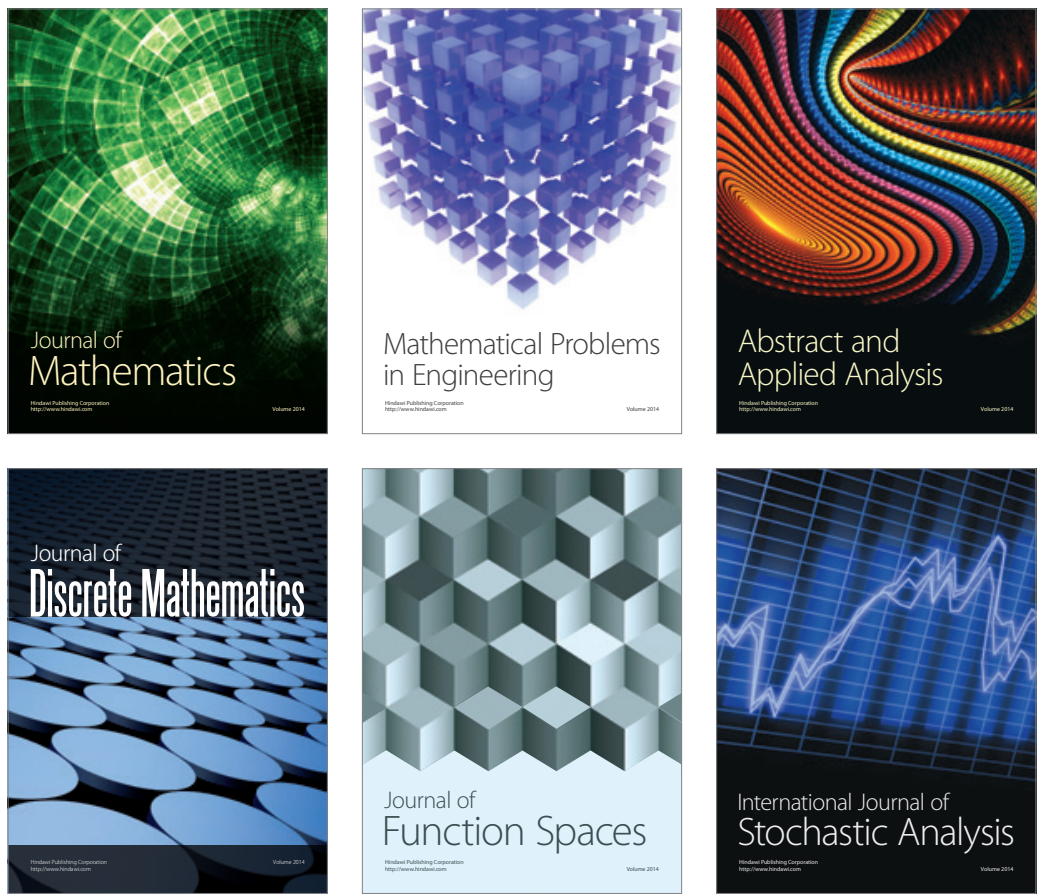

Journal of

Function Spaces

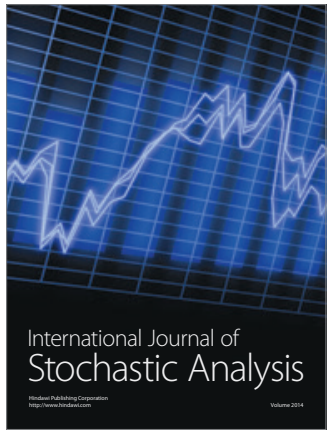

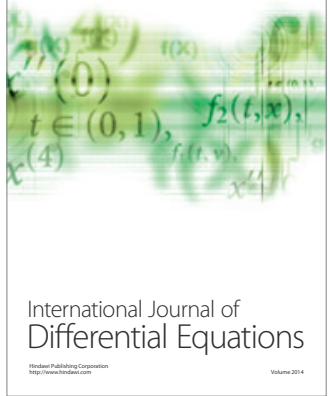
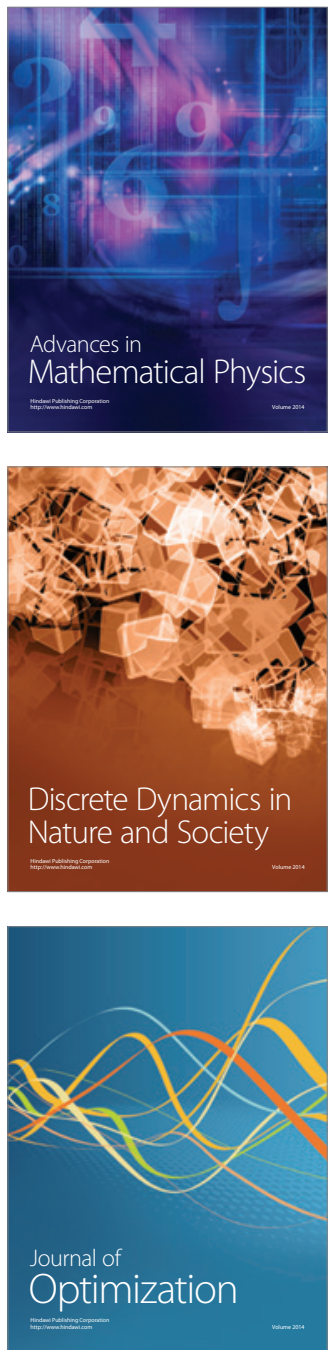